\title{
FORTALEZAS Y ÁREAS DE MEJORA DE LA FORMACIÓN INICIAL DOCENTE EN LECTOESCRITURA EN LA REPÚBLICA DOMINICANA
}

\author{
Strengths and Areas for Improvement of Preservice \\ Teacher Education in Early Literacy in the Dominican Republic
}

Liliana Montenegro-de-Olloqui ${ }^{1}$

Imontenegro@pucmm.edu.do

${ }^{1}$ Pontificia Universidad Católica Madre y Maestra, República Dominicana

Fecha de recepción: 4/05/2020

Fecha de aprobación: 15/6/2020

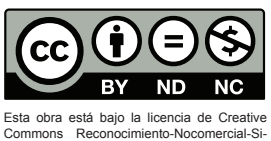

ISSN (impreso): 2636-2139

ISSN (en línea): 2636-2147

Sitio web: https://revistas.isfodosu.edu.do/recie

\section{Resumen}

Este artículo enfoca la formación en lectoescritura inicial (LEI) de estudiantes de la licenciatura en Educación Primaria Primer Ciclo de una institución de educación superior de la República Dominicana, privilegiando el análisis curricular. La investigación, de corte cualitativo, buscó analizar: 1) la alineación del currículo de formación con la evidencia investigativa; 2) las fortalezas y las brechas del plan de estudios; y 3) las diferencias entre el currículo prescrito y el aplicado en las aulas. Los datos se recolectaron mediante el mapeo de 25 cursos, entrevistas con seis docentes formadoras y doce docentes en formación en las tres sedes de la carrera, y nueve observaciones de aula. Los hallazgos indican que el plan de estudios está alineado con la literatura científica sobre la enseñanza de la LEI, aunque algunos ámbitos analizados no se abordan en profundidad (vocabulario, conciencia fonológica, decodificación y fluidez lectora). Se detectaron fortalezas: prescripción clara del enfoque teórico para la enseñanza (constructivista) y para la LEI (procesual cognitivo y comunicativo); prácticas en contextos reales para el cuerpo de docentes en formación, y brechas: diferencias entre las sedes sobre la formación en LEI de algunos profesores; escaso dominio del estudiantado sobre la evaluación del aprendizaje y ausencia de consulta sistemática de la bibliografía. Se constataron diferencias entre el currículo prescrito y el aplicado respecto al peso de la teoría y la práctica, al modelado, al enfoque de la enseńanza de la LEI y a la selección, elaboración y utilización de materiales para su instrucción.

Palabras clave: educación superior, formación inicial docente, investigación curricular, lectoescritura.

\section{Abstract}

This article focuses on the pre-service teacher education in Early Grade Literacy (EGL) in the First Cycle Primary Education Bachelor's degree at a higher education institution in the Dominican Republic, prioritizing curricular analysis. The research, of a qualitative nature, sought to analyze: 1) the alignment of the training curriculum in relation to current evidence; 2) strengths and gaps of the curriculum; and 3) the differences between the prescribed and applied curriculum in classrooms. The data was collected through the mapping of 25 courses, interviews with six teacher educators, 12 preservice teachers, in the three sites of the Institute, and nine classroom observations. The findings indicate that the curriculum is aligned with the current evidence for teaching EGL, although some areas analyzed are not addressed in-depth (vocabulary, phonological awareness, decoding and reading fluency). Strengths were detected: clear prescription of the theoretical approach for teaching (constructivism) and for EGL (cognitive and communicative approaches); practices in real contexts for teachers in training and gaps: differences between sites on EGL training for some teachers; low students mastery of learning assessment and lack of systematic consultation of the bibliography. Differences were found between what is prescribed and applied curricula regarding the weight of theory and practice, modeling, the approach to teaching the EGL, and the selection, development, and use of materials for teaching.

Keywords: curriculum research, early literacy, higher education, pre-service teacher education. 


\section{Introducción}

Este artículo presenta los resultados de una investigación que estudió la formación docente en el ámbito de la lectoescritura inicial (LEI) que reciben estudiantes de la licenciatura en Educación Primaria Primer Ciclo, impartida por una institución de educación superior de la República Dominicana. El estudio forma parte de una propuesta regional desarrollada por la Red para la Lectoescritura Inicial de Centroamérica y el Caribe (RedLEI).

Las premisas teóricas de la investigación mostraron la estrecha relación entre el dominio de determinados conocimientos y habilidades y las buenas prácticas en la enseñanza de la lectura y escritura en el nivel inicial (Chesterfield \& Abreu-Combs, 2011; Ferreiro et al., 2002; Kaufman et al., 2015; McEwan, 2015; Moats, 2009; Rivkin et al., 2005). Asimismo, el equipo partió del entendido de que el cuerpo docente es el factor clave de la calidad de la enseñanza de la LEI y, por tanto, es fundamental asegurarse de que se preparen como especialistas de este campo (Bellei et al., 2013; Bruns \& Luque, 2015).

El trabajo, de corte cualitativo, buscó responder las siguientes preguntas de investigación:

1. ¿Cómo se alinea el currículo de formación inicial docente con la evidencia sobre cómo se aprende la lectoescritura en los primeros ańos y qué debe saber y poder hacer el cuerpo docente para una instrucción efectiva?

2. ¿Cuáles son las fortalezas y las brechas del plan de estudios de formación inicial docente en relación con la evidencia investigativa?

3. ¿En qué se diferencia el currículo prescrito del currículo realmente aplicado en los programas de formación inicial docente en el ámbito de la lectoescritura inicial?

Se espera que, a partir de los resultados, se emprendan iniciativas de reflexión y cambio en la licenciatura en Educación Primaria Primer Ciclo de la República Dominicana, así como en las políticas curriculares y de formación continua del cuerpo docente a nivel nacional y regional. Asimismo, que las niñas y los niños de la región consigan ventanas de oportunidad, que solo pueden abrirse desde las experiencias significativas que puedan tener a través del dominio de las habilidades complejas de la lectura y la escritura.

Respecto al contexto, cabe destacar que, en la República Dominicana, la gran mayoría de estudiantes de tercer grado presentan dificultades en la comprensión lectora. El Tercer Estudio Regional Comparativo y Explicativo (TERCE) de la UNESCO (2010) concluyó que el estudiantado de este grado solo es capaz de comprender cierta información explícita y hacer inferencias simples en determinados textos, y tiene dificultades para analizar, comparar e interpretar información implícita más compleja. Asimismo, la evaluación diagnóstica nacional de tercer grado de primaria identificó que "solo el $12 \%$ de los estudiantes de tercer grado demostró las competencias específicas establecidas en el diseño curricular nacional para ese grado en Lengua Española y el 50\% alcanzó el nivel elemental, evidenciando importantes rezagos en sus aprendizajes" (MINERD, 2017, p. 9).

Con relación a la formación inicial docente, el actual sistema nacional tiene su base en la Ordenanza 05/97 que dio lugar a la creación y puesta en funcionamiento del Instituto Nacional de Formación y Capacitación del Magisterio (INAFOCAM). Mediante la Ordenanza 01-2004 se logró consensuar un nuevo plan de estudio y elevar las escuelas normales a Instituto Superior de Formación Docente (Decreto 427-00, 2000). Para ejercer la profesión docente es obligatorio contar con una licenciatura emanada de una institución superior.

El Ministerio de Educación Ciencia y Tecnología (MESCyT) propuso, mediante la Normativa para la Formación Docente de Calidad en la República Dominicana (MESCyT 09-2015), el rediseño de los planes de estudio, para que quienes egresaran del sistema adquirieran los conocimientos y las competencias que aseguraran al estudiantado el logro de aprendizajes significativos. En virtud de la misma normativa, la acreditación de los programas y la 
prueba de ingreso para aspirantes devinieron obligatorias, entre otros requisitos.

Estas normas han posibilitado una mayor coherencia en la oferta formativa de las carreras de educación, con planes de estudio organizados según competencias generales y específicas. Respecto al primer ciclo del nivel primario, establecen que quien ejerce la docencia debe dominar los procesos de enseñanza-aprendizaje para la lectoescritura y el razonamiento matemático de niños y niñas de 6 a 9 años de edad y conocer ampliamente las características psicológicas y del proceso de desarrollo de esta etapa de la vida. En cuanto a la estructura curricular, establecen 160 créditos para el programa distribuidos por componentes: formación general (15\%), psicopedagógica $(50 \%)$ y disciplinaria $(35 \%)$.

\section{Revisión de la literatura}

Estudios recientes (Andrade-Calderón, et al., 2020; Litt et al., 2015) demuestran que, para enseñar la lectoescritura inicial (LEI) en los primeros grados y contribuir con la calidad de la formación del estudiantado, el cuerpo docente debe dominar conocimientos teóricos (saber) y prácticos (saber hacer) en relación con tres dimensiones: el desarrollo infantil, el conocimiento de la LEI y la evaluación de los aprendizajes, incluyendo el uso de los resultados para regular la práctica y colaborar con las políticas educativas.

\subsection{Dimensión del desarrollo infantil}

Se refiere al conocimiento sobre la evolución de la niñez para poder apoyarla en su trayectoria de desarrollo de manera efectiva (Copple \& Bredekamp, 2009; Darling-Hammond \& Bransford, 2005). Por otro lado, el profesorado en formación necesita conocer cómo se desarrollan los procesos cognitivos y las prácticas sociales culturalmente contextualizadas (Bruning \& Kauffman, 2015), para contar con herramientas que incidan en el aprendizaje de la LEI. De acuerdo con la evidencia consultada, esta dimensión abarca diez ámbitos clave para la formación de los futuros docentes, que se describen a continuación.
Teorías del desarrollo. El conocimiento de los procesos cognitivos y socioculturales (Bezemer \& Kress, 2010) que se realizan para apropiarse de la lengua escrita (Díaz et al., 2015) aportará a los formadores un marco referencial para influir efectivamente en la enseñanza de la lectura y la escritura (Manghi, 2011).

Teorías del aprendizaje y la motivación. La comprensión profunda del desarrollo del lenguaje, su relación con la lectura y la escritura, y el conocimiento de paradigmas sobre cómo se aprende y cómo se motiva al estudiantado son fundamentales para tomar decisiones educativas de manera efectiva (Fons, 2004; ILA, 2018; Sáez, 2015).

Desarrollo del lenguaje. El cuerpo docente necesita tener conocimientos sobre la estructuración y el desarrollo del lenguaje y la complejidad de este proceso para ser más efectivo en el aula (Adger et al., 2018; Hoyle \& Adger, 1998; Wong-Fillmore \& Snow, 2000). También necesita conocer las estrategias necesarias para intervenir certeramente en el proceso de adquisición de la lengua (Avendaño \& Miretti, 2006), teniendo en cuenta la diversidad de habilidades de la niñez (Menyuk \& Brisk, 2005).

Fundamentos de la enseñanza-aprendizaje de la lectura. Se refiere al conocimiento por parte de los formadores de las diversas hipótesis que desarrollan los niños en la lectura inicial, la relación entre componentes de nivel superior e inferior que intervienen en el proceso (Hoover \& Gough, 1990) y cómo desarrollar en el estudiantado la resolución de problemas que les permitan avanzar como lectores, entendiendo que las diferentes prácticas de lectura conviven en la cultura y necesitan ser enseńadas y aprendidas en la escuela (Kaufman et al., 2015).

Fundamentos de la enseñanza-aprendizaje de la escritura. Con el propósito de ayudar al alumnado a desarrollar competencias en el uso de lengua escrita (Berman, 2004; Cassany, 2012), el cuerpo docente necesita conocer las diferentes etapas de adquisición de la escritura inicial, así como saber que, para escribir bien, se deben dominar procesos cognitivos (Alamargot \& Chanquoy, 2001) en los que intervienen diferentes niveles de conocimiento lingüístico: fono- 
lógico, morfosintáctico, léxico, pragmático y discursivo (Salgado, 2000).

Neurociencia de la LEI. Para el cuerpo docente resulta muy valioso tener conocimientos sobre la relación del cerebro con la lectoescritura (Brem et al., 2010) para orientar mejor su aprendizaje y apoyar a quienes presentan alguna dificultad durante este proceso (Leppänen et al., 2010). Además, podrá comprender cómo la lectoescritura estimula el desarrollo cognitivo del alumnado (Casey et al., 2002; Liston et al. 2005) y cómo la lectura refuerza la respuesta cerebral, creando nuevos circuitos y conexiones neuronales (Goldman-Rakic, 1987; McLaren et al., 2012; Olesen et al., 2004).

Psicología y sociología de la lectoescritura. La alfabetización implica la entrada a una comunidad discursiva en la que la lectura y la escritura están inscritas en prácticas sociales (Bruning \& Kauffman, 2015). Estas prácticas permiten dar sentido al aprendizaje de la lectura, el género, la escritura, el contenido y la audiencia (Bazerman et al., 2005) y direccionalidad a las trayectorias del alumnado (Barletta-Manjarres et al., 2012).

Impacto del contexto en el aprendizaje de la LEI. Es preciso que los formadores desarrollen competencias sobre los usos sociales de la lengua escrita para poder ofrecer una enseñanza ajustada a las necesidades del estudiantado, a su contexto (Ferreiro \& Teberosky, 1979; Teberosky, 2000) y a sus tradiciones lingüísticas (Lucas \& Grinberg, 2008).

Bilingüismo. Para el cuerpo docente alfabetizador es importante tener conocimientos sobre el bilingüismo (Romaine, 1995), pues puede presentársele, entre otros, el caso de estudiantes cuya lengua materna no sea el espańol (Ortega, 2008). Diversas investigaciones indican que el desarrollo de la primera lengua ayuda al aprendizaje de la segunda (August \& Shanahan, 2006; Genesee et al., 2006). Por otra parte, quienes asisten a programas bilingües (G. McField \& D. McField, 2014; Rolstad et al., 2005) avanzan académica y lingüísticamente a largo plazo y su organización cognitiva y conceptual se ve favorecida (Bialystok, 2011).
Trastornos del desarrollo de la lectura y escritura. En el nivel primario, las dificultades de aprendizaje de la lectoescritura representan un considerable porcentaje dentro de las dificultades de aprendizaje en general (Asociación Americana de Psiquiatría, 2013) e impactan en el alumnado en los otros niveles de su formación. Según Tunmer \& Hoover (2019), el cuerpo docente debe conocer las capacidades cognitivas subyacentes al aprendizaje de la lectoescritura para poder comprender las dificultades que se producen en el curso de su adquisición, prevenir su aparición y favorecer su aprendizaje efectivo.

\subsection{Dimensión del conocimiento de la LEI}

Quienes enseñan la LEI eficientemente son capaces de preparar al estudiantado para comprender ideas complejas, desarrollar su criticidad, sintetizar información de diferentes fuentes y usar la lectura para transformar el conocimiento, además de comprender cómo se aprende y brindar el apoyo necesario para que sus estudiantes triunfen (National Research Council, 2010). Los once ámbitos de esta dimensión que se describen a continuación son fundamentales en la formación docente porque proveen conocimientos teóricos sobre los procesos de la lectura y la escritura (Shanahan, 2015) y conocimientos prácticos sobre cómo enseñarlos (Solé, 2009).

Lenguaje oral. Promover el uso del lenguaje oral permite que el estudiantado organice su pensamiento y desarrolle su cognición, lo que repercute en la comprensión lectora y la expresión escrita. Conocer las particularidades del lenguaje oral (Ferreiro et al., 2002) es necesario para el cuerpo docente alfabetizador (Stahl et al., 2009), puesto que proporciona la base para el desarrollo de la LEI (American Speech-Language-Hearing Association [ASHA], 2008).

Concepto de lo impreso. El cuerpo docente alfabetizador debe conocer el funcionamiento de los materiales impresos (Holdgreve-Resendez, 2010) para poder enseñarlo: diferenciación entre dibujos y letras, direccionalidad de izquierda a derecha y de arriba abajo para la lectura y la escritura en lengua espańola, comprensión de "la naturaleza del objeto de cono- 
cimiento involucrado en este aprendizaje" (Ferreiro, 1997, p. 13), en este caso, el sistema alfabético de escritura.

Conciencia fonológica. Según Bravo et al., (2011), es una habilidad metalingüística que se desarrolla progresivamente durante los primeros ańos de vida y permite tomar conciencia de las unidades más grandes y concretas del habla (palabras y sílabas) hasta las más pequeñas y abstractas (fonemas). Su meta final es lograr establecer la relación fonema-grafema. Tiene un papel crucial en la adquisición y desarrollo de la lectura y la escritura (Arancibia et al., 2012; Defior \& Serrano, 2011; Sastre-Gómez et al., 2017). Representa uno de los mejores "predictores de escolarización y de cuán bien los niños y las niñas aprenderán a leer durante los primeros dos años de instrucción" (National Reading Panel [NRP], 2000, p. 7).

Principio alfabético. Aprender la lengua escrita necesita de la intervención de profesionales expertos que puedan enseñar al alumnado, de manera intencional, que existen relaciones sistemáticas entre letras y sonidos (Ferreiro 2002), además de conocer la estructura de la lengua y el desarrollo del proceso de lectura; igualmente requiere poner en práctica una metodología que permita diferenciar la instrucción para estudiantes diversos (Moats, 2009).

Vocabulario. Una parte esencial de la habilidad comprensiva radica en el conocimiento del significado de las palabras. Esta habilidad está conectada con el conocimiento general del sujeto y con la capacidad de resolución de problemas. Por estas razones es necesario prestar atención a la enseńanza explícita e intencional del vocabulario en el contexto de los programas de alfabetización (NICHD, 2000).

Fluidez lectora. El cuerpo docente alfabetizador necesita conocer cómo se desarrollan las capacidades para leer un texto con precisión, velocidad y entonación adecuada. Según De Mier et al. (2012), en la fluidez confluyen las habilidades de dos subprocesos de la lectura: el reconocimiento de palabras y la comprensión.
Comprensión lectora. Representa la finalidad última del aprendizaje de la lectura. Es el conocimiento de cómo se desarrolla el proceso de interacción entre el lector y el texto, para darle significado y entenderlo (Solé, 2009), coordinando informaciones que provienen de diferentes fuentes: el texto, el contexto y el conocimiento previo. Se enseña mediante estrategias (Camargo et al., 2013) que permitirán al alumnado la planificación de la tarea general de lectura y su motivación, la realización de inferencias de distinto tipo, la comprobación, revisión y control de lo que se lee y la toma de decisiones según los objetivos.

Conexiones entre lectura y escritura. El cuerpo docente debe saber que la lectura y la escritura son procesos cognitivos de gran complejidad e interconectados (Graham \& Hebert, 2010; Shanahan, 2015), cuyo aprendizaje se caracteriza por la internalización de reglas a través de la formación de hipótesis y confrontación. Se trata de sistemas simbólicos de representación, y ambos tienen propiedades específicas (Ferreiro \& Gómez, 1986).

Grafomotricidad. Es el conocimiento y análisis de los aspectos cognitivos, afectivos y psicomotores que intervienen en la realización de las grafías (Alviz, 2012).

Expresión escrita. Según Condemarín \& Medina (2000), la escritura demanda un dominio progresivo de complejas operaciones cognitivas. Se trata del conocimiento de cómo se enseña y se aprende la capacidad de expresar ideas por escrito cuya ejecución depende — según Hayes y Flower (1980) y Hayes (1996) — de la activación de la planificación, textualización y revisión como procesos intelectuales interactivos y recursivos (Bui et al., 2006), que se monitorean y regulan mediante la conciencia metacognitiva del escritor (Sitko, 1998). Gallego et al. (2014) sostienen que la autorregulación requiere ayuda gradual por parte del cuerpo docente.

Convenciones de la escritura. Se trata de que el cuerpo docente comprenda que la habilidad de leer y escribir tiene relación con la sintaxis, es decir, la estructura gramatical de la lengua (Casado, 2000; Faigley $\&$ Witte, 1981) y la ortografía, o sea, las reglas de escri- 
tura, puntuación y uso de mayúsculas (Berninger $\&$ Amtmann, 2003; Salgado, 2000).

\subsection{Dimensión de la evaluación}

Se trata del conocimiento y saber hacer que el cuerpo de docentes en formación necesita dominar y utilizar para evaluar las habilidades de lectura y escritura de sus estudiantes, los recursos didácticos seleccionados y la adecuación de la planificación de las clases al contexto. Incluye además el conocimiento sobre el uso de los resultados de las evaluaciones para la toma de decisiones dentro del aula y la mejora de la práctica docente (S. Chappuis \& J. Chappuis, 2008). Es fundamental que los docentes en formación conozcan qué evaluación utilizar para cada resultado deseado y por qué utilizar un instrumento de evaluación en particular. Según la evidencia investigativa, esta tercera dimensión comprende cuatro ámbitos que habilitan al docente alfabetizador para evaluar la adquisición de la lectoescritura inicial.

Propósito y tipos de evaluación. Según Condemarín y Medina (2000), el profesorado alfabetizador necesita conocer el carácter multidimensional de la evaluación como parte esencial del proceso de enseñanza-aprendizaje. Asimismo, debe dominar un amplio repertorio de procedimientos, técnicas e instrumentos evaluativos, teniendo en cuenta la complejidad de la adquisición de la lengua escrita y la heterogeneidad del alumnado (McKenna \& Stahl, 2009).

Diseño de evaluaciones formativas. El profesorado necesita conocer que la evaluación fortalece los procesos formativos y de autorregulación de la enseñanza-aprendizaje, por lo que debe incluirla en las diversas propuestas didácticas (evaluación formativa) y no solo aplicarla al final, como medición de resultados (evaluación sumativa). Perrenoud (2008, p. 136) la llama observación formativa y afirma que "permite guiar y optimizar los aprendizajes en curso, sin preocuparse por clasificar, certificar, ni seleccionar”.

Aplicación de evaluaciones formativas. Según los Estándares para la preparación de profesionales de la alfabetización 2017 (ILA, 2018), el cuerpo docente necesita conocimiento para poder usar herramientas de evaluación apropiadas para evaluar, diagnosticar y medir el logro de alfabetización de los estudiantes, así como informar la instrucción y evaluar las intervenciones.

Interpretación y uso de los resultados de la evaluación. Es el conocimiento que permite al cuerpo docente saber que cada vez que aplica una evaluación, tiene la posibilidad de obtener información valiosa sobre el proceso de aprendizaje de sus estudiantes y así tomar decisiones pertinentes para adecuar la instrucción. Dado que la aplicación de este conocimiento tiene lugar durante el desarrollo de la enseñanza, también permite que el alumnado pueda usar sus resultados para regular su aprendizaje (S. Chappuis \& J. Chappuis, 2008).

\section{Metodología}

El objetivo de esta investigación fue conocer los procesos de formación inicial docente para la instrucción de la LEI en el nivel primario, y formular recomendaciones para asegurar la alineación entre lo que dice la evidencia investigativa que el cuerpo docente necesita saber y ser capaz de hacer y lo que realmente se les enseña en las instituciones formadoras.

Se diseñó una investigación regional con enfoque cualitativo que buscó responder a las tres preguntas de investigación ya mencionadas. De marzo a junio del 2018, el equipo de investigadores sénior y especialistas en LEI realizaron una revisión de la literatura para identificar la evidencia investigativa más reciente sobre los conocimientos y metodologías requeridos para enseñar a leer y escribir. A partir de esta revisión, se construyó el marco conceptual de la investigación, al que, al momento de reproducir la investigación en la República Dominicana, se agregaron algunas referencias hispanoamericanas. Los detalles del diseño, instrumentos de recolección de datos y análisis de la investigación regional pueden consultarse en https://red-lei.org/nuestro-equipo/investigacion-regional/.

Para llevar a cabo la recolección y el análisis de datos para la República Dominicana (octubre de 2019 
a marzo de 2020) se desarrollaron tres tipos de actividades principales: 1) mapeo de los currículos de formación inicial docente en el ámbito de la LEI considerando los marcos conceptuales derivados de la evidencia internacional; 2) entrevistas con formadores de docentes (DF) y docentes en formación (DeF); y 3) observaciones de aulas en las que tiene lugar la formación docente. Se procuró que, al triangular los datos de las diferentes fuentes, la investigación ofreciera una imagen de qué se enseña al grupo de DeF para enseñar la LEI, y se obtuviera información para analizar el grado en que tal formación se acerca o aleja de lo que la evidencia sugiere.

\subsection{Unidad de estudio}

Para realizar el componente de la República Dominicana de esta investigación regional, se seleccionó como unidad de estudio una institución de educación superior que estuviera impartiendo la licenciatura en Educación Primaria Primer Ciclo en más de una sede. La licenciatura tiene una duración de tres ańos y ocho meses, distribuidos en 11 cuatrimestres. El programa de estudio se compone de un total de 53 asignaturas organizadas en tres bloques: 1) Formación general (24 créditos); 2) Formación psicopedagógica (79 créditos); y 3) Formación disciplinar (57 créditos); lo que arroja un total de 160 créditos. Cabe señalar que la formación disciplinar incluye nueve asignaturas de Lectoescritura, cinco asignaturas de Matemática y cuatro asignaturas de Ciencias
Sociales y Naturales, todas enfocadas en el primer ciclo de primaria.

Después de recibir la autorización de la institución de educación superior del país seleccionada, se confirmó que dicha licenciatura se estaba impartiendo en tres sedes. Al momento de la implementación de la investigación, en una de ellas se estaba cursando el décimo cuatrimestre; en otra, el séptimo; y en la tercera, el quinto cuatrimestre.

A partir de las preguntas de investigación y la definición del marco conceptual, se diseñaron los instrumentos de investigación, a saber, una guía para el mapeo de los currículos, una guía semiestructurada de entrevistas para DF y para DeF y una guía de observaciones de aula. En el caso de las entrevistas, estas se realizaron de manera individualizada y se registraron en grabaciones de audio. Los resultados se almacenaron en una base de datos nacional con formato digital.

La investigación se adhiere a los principios éticos para la investigación en seres humanos. El Comité de Ética del American Institutes for Research (AIR) revisó el protocolo de investigación y dio el aval regional para su implementación.

\subsection{Recopilación de datos}

El trabajo de campo se realizó de septiembre de 2019 a febrero de 2020. A continuación se presentan las fuentes, muestra y métodos de recolección de datos (Tabla 1). 
Tabla 1. Fuentes, muestra y métodos de recolección de datos

\begin{tabular}{|c|c|c|c|}
\hline \# Preguntas de investigación & Fuente & Muestra & Método de recolección \\
\hline $\begin{array}{l}\text { 1. ¿Cómo se alinea el currículo de } \\
\text { formación inicial docente con la } \\
\text { evidencia sobre cómo se aprende } \\
\text { la lectoescritura en los primeros } \\
\text { años y qué debe saber y poder } \\
\text { hacer el cuerpo docente para una } \\
\text { instrucción efectiva? }\end{array}$ & $\begin{array}{l}\text { Cursos pertinentes } \\
\text { del programa de } \\
\text { formación selec- } \\
\text { cionado }\end{array}$ & 25 & $\begin{array}{l}\text { La investigadora recolectó lo que es- } \\
\text { taba disponible públicamente y luego } \\
\text { solicitó documentos adicionales a la } \\
\text { institución seleccionada. }\end{array}$ \\
\hline \multirow[t]{3}{*}{$\begin{array}{l}\text { 2. ¿Cuáles son las fortalezas y } \\
\text { las brechas del plan de estudios } \\
\text { de formación inicial docente en } \\
\text { relación con la evidencia investi- } \\
\text { gativa? }\end{array}$} & $\begin{array}{l}\text { Cursos pertinentes } \\
\text { del programa de } \\
\text { formación selec- } \\
\text { cionado }\end{array}$ & 25 & \multirow{3}{*}{$\begin{array}{l}\text { Además de la recolección de datos dis- } \\
\text { ponibles, la investigadora obtuvo datos } \\
\text { en la observación del aula, entrevistas } \\
\text { con docentes formadores y en forma- } \\
\text { ción, y fichas técnicas del personal } \\
\text { administrativo. }\end{array}$} \\
\hline & $\begin{array}{l}\text { Entrevistas con } \\
\text { docentes forma- } \\
\text { dores }\end{array}$ & 6 & \\
\hline & $\begin{array}{l}\text { Entrevistas con } \\
\text { docentes en for- } \\
\text { mación }\end{array}$ & 12 & \\
\hline \multirow{3}{*}{$\begin{array}{l}\text { 3. ¿En qué se diferencia el currí- } \\
\text { culo prescrito del currículo real- } \\
\text { mente aplicado en los programas } \\
\text { de formación inicial docente en el } \\
\text { ámbito de la lectoescritura inicial? }\end{array}$} & $\begin{array}{l}\text { Entrevistas con } \\
\text { docentes forma- } \\
\text { dores }\end{array}$ & 6 & $\begin{array}{l}\text { Uno a uno con los seis docentes forma- } \\
\text { dores participantes en la investigación. }\end{array}$ \\
\hline & $\begin{array}{l}\text { Entrevistas con } \\
\text { docentes en for- } \\
\text { mación }\end{array}$ & 12 & $\begin{array}{l}\text { Uno a uno con los doce docentes parti- } \\
\text { cipantes en la investigación. }\end{array}$ \\
\hline & $\begin{array}{l}\text { Observaciones del } \\
\text { aula }\end{array}$ & 9 & $\begin{array}{l}\text { Observaciones del período completo } \\
\text { de los cursos relacionados con la LEl. }\end{array}$ \\
\hline
\end{tabular}

\subsection{Análisis de datos}

Se realizaron dos talleres en septiembre y diciembre de 2019, el primero para discutir el procedimiento de implementación de la investigación y el segundo para el uso del software MAXQDA para análisis cualitativo. Después de concluir el mapeo, las entrevistas y observaciones, y la digitación de todos los datos, se revisó el árbol de categorías elaborado a partir del marco conceptual que luego fue utilizado para la codificación del material recolectado.
El primer árbol de categorías, propuesto por el equipo de coordinación y asesoría de la investigación, fue revisado y validado con el grupo de investigadores. Después de considerar la propuesta, se agregaron ámbitos complementarios a cada dimensión y categorías que se denominaron "generales" porque no se correspondían directamente con los ámbitos de las tres dimensiones del marco conceptual, pero ofrecían información que enriquecía el análisis. En el caso de la Dimensión 3, se modificaron los ámbitos propuestos originalmente, tal como puede observarse en la Figura 1. 
MONTENEGRO-DE-OLLOQUI

Fortalezas y áreas de mejora de la formación inicial docente en lectoescritura...

Figura 1. Árbol de categorías para análisis de datos por dimensiones

\begin{tabular}{|c|c|c|}
\hline Dimensión 1. Desarrollo infantil & $\begin{array}{l}\text { Dimensión 2. Conocimiento de } \\
\text { la LEI }\end{array}$ & Dimensión 3. Evaluación \\
\hline $\begin{array}{l}\text { Teorías del desarrollo } \\
\text { Teorías del aprendizaje y motiva- } \\
\text { ción } \\
\text { Desarrollo del lenguaje } \\
\text { Fundamentos de la enseñan- } \\
\text { za-aprendizaje de la lectura } \\
\text { Etapas del aprendizaje de la lec- } \\
\text { tura* } \\
\text { Fundamentos de la enseñan- } \\
\text { za-aprendizaje de la escritura } \\
\text { Etapas del aprendizaje de la escri- } \\
\text { tura* } \\
\text { Neurociencia de la LEI } \\
\text { Psicología y sociología de la lecto- } \\
\text { escritura } \\
\text { Impacto del contexto en el aprendi- } \\
\text { zaje de la LEl } \\
\text { Bilingüismo } \\
\text { Trastornos del desarrollo de la lec- } \\
\text { tura y escritura }\end{array}$ & $\begin{array}{l}\text { Concepto de lo impreso } \\
\text { Conciencia fonológica } \\
\text { Principio alfabético } \\
\text { Vocabulario } \\
\text { Fluidez lectora } \\
\text { Comprensión lectora } \\
\text { Lenguaje oral } \\
\text { Conexiones entre lectura y escri- } \\
\text { tura } \\
\text { Grafomotricidad } \\
\text { Expresión escrita } \\
\text { Convenciones de la escritura } \\
\text { Habilidades implicadas en LEl* } \\
\text { Cultura lectora* }\end{array}$ & $\begin{array}{l}\text { (Versión original) } \\
\text { Propósito y tipos de evaluación } \\
\text { Diseño de evaluaciones formativas } \\
\text { Aplicación de evaluaciones forma- } \\
\text { tivas } \\
\text { Interpretación y uso de los resulta- } \\
\text { dos de la evaluación } \\
\text { (Versión final) } \\
\text { Propósitos de la evaluación } \\
\text { Tipos de evaluación } \\
\text { Evaluación de los aprendizajes* } \\
\text { Diseño de herramientas de evalua- } \\
\text { ción } \\
\text { Aplicación de herramientas de eva- } \\
\text { luación } \\
\text { Interpretación y uso de los resulta- } \\
\text { dos de la evaluación }\end{array}$ \\
\hline
\end{tabular}

Fuente: Andrade-Calderón, Stone \& Vijil, 2020.

*Ámbitos complementarios.

\subsubsection{Definición de ámbitos complementarios}

- Etapas del aprendizaje de la lectura. Cada uno de los procesos graduales de apropiación de la comprensión de la lengua escrita.

- Etapas del aprendizaje de la escritura. Cada uno de los procesos de apropiación de la escritura hasta llegar a la convencional, que refleja una cierta concepción de la niñez respecto a lo que es la escritura y a cómo se ejecuta (Ferreiro \& Teberosky, 1979).

- Habilidades implicadas en LEI. Es el conocimiento de las habilidades cognitivas relacionadas con la enseñanza y el aprendizaje de la LEI.

- Cultura lectora. Prácticas sociales relacionadas con la promoción de la lectura en diferentes soportes y con la comprensión y utilización de la información escrita para la búsqueda del conocimiento, información o entretenimiento.
- Evaluación de los aprendizajes. Es un componente del proceso educativo que permite observar, recoger y analizar la información obtenida respecto de las posibilidades, necesidades y logros del estudiantado, para mejorar el proceso de enseñanza-aprendizaje a partir de la reflexión, la emisión de juicios de valor y la toma de decisiones pertinentes y oportunas.

\subsubsection{Definición de categorías generales}

- Enfoque teórico. Fundamentos teóricos de carácter epistemológico, pedagógico y psicológico que sustentan el proceso de enseñanza-aprendizaje de la LEI.

- Prácticas pedagógicas. Según Duque et al. (2013, p. 17), se trata de "las variadas acciones que el docente ejecuta para permitir el proceso de formación integral en el estudiante”.

- Medios y recursos didácticos. Componentes mediadores que ayudan al cuerpo docente en 
su tarea de enseñar y que facilitan al alumnado el logro de los objetivos de aprendizaje.

- Práctica para los docentes en formación. La práctica constituye una "entidad coherente e interdependiente dentro del currículum de formación docente" (Sayago \& Chacón, 2006, p. 57) que posibilita al practicante ir construyendo su identidad como docente al enfrentarse a situaciones reales en las que usa herramientas conceptuales, procedimentales y actitudinales para observar, intervenir, reflexionar, reconstruir y valorar realidades en su complejidad.

- Supervisión y acompañamiento. El cuerpo docente en formación es acompañado en el aula para asistir y potenciar el éxito de su intervención pedagógica, con el propósito de mejorar el desempeño del estudiantado. Este monitoreo pedagógico favorece la reflexión de su gestión en el aula, la articulación teoría-práctica y el fortalecimiento de su saber al compartir la experiencia entre pares (Martínez \& Montenegro, 2010). También permite asesorarlo y capacitarlo según los resultados (Regalado et al., 2016).

- Formas de evaluar el aprendizaje. Obtención de evidencias sobre lo aprendido para poder juzgar el progreso del estudiantado e implementar mejoras.

- Estándares nacionales. Marco regulatorio del sistema educativo para asegurar un mayor control de la calidad, basado en estándares, así como en la evaluación y rendición de cuentas de los resultados alcanzados por el estudiantado y del desempeño del profesorado, de directivos y administradores (UNICEF, 2010).

- Acceso al currículo nacional que se espera que enseñe. Se trata de incorporar en el saber de los futuros docentes el conocimiento y la opción de consulta del currículo nacional vigente (MINERD, 2016), que expresa los saberes, valores y competencias que debe lograr el estudiantado en el primer ciclo del nivel primario.

- Tiempo previsto para la enseñanza del conocimiento de LEI. Según los programas analizados, se refiere al número de horas asignadas para la enseńanza de la LEI.

- Tiempo real dedicado a la enseñanza del conocimiento de la LEI. Según las entrevistas y observaciones realizadas, se trata del número de horas que el cuerpo docente formador o en formación declara que se dedicaron para la enseñanza de la LEI.

- Bibliografía. Conjunto de referencias sobre publicaciones en libros, documentos, revistas, información de internet, películas, emisiones de radio o televisión, discos multimedia, etc. (Patiño, 2005).

- Perfil del docente formador. Conjunto de conocimientos, habilidades y actitudes requeridas para desarrollar la labor de formación de otros docentes, de acuerdo con criterios valorativos y parámetros de calidad de una institución determinada.

Al tener toda la información codificada, se recuperaron los segmentos de texto para realizar el análisis de tipo categorial que permitió responder las preguntas de investigación. Para la primera pregunta se utilizó la información del mapeo de los programas y syllabus de los cursos de la licenciatura, tanto los especializados como los psicopedagógicos relacionados con la LEI. Esa información se codificó en las categorías relacionadas directamente con las tres dimensiones del marco conceptual del estudio. La triangulación de datos de diferentes fuentes permitió responder las preguntas segunda y tercera. Para lograrlo, se analizó la información obtenida del mapeo de los programas y syllabus de los cursos, las entrevistas y observaciones en el aula organizadas en categorías relacionadas directamente con las tres dimensiones del marco conceptual, y con las categorías complementarias y generales. 


\section{Resultados}

\subsection{Pregunta 1}

El plan de estudio se encuentra alineado con la evidencia científica sobre la enseñanza de la LEI, pues incluye la mayor parte de los ámbitos considerados en las tres dimensiones (26 de 27). Solamente el bilingüismo no fue contemplado por el programa de formación, aunque el Gobierno dominicano garantiza el libre acceso a la educación de la niñez, sin importar su nacionalidad, en cumplimiento de una política de Estado de equidad e inclusión social.

\subsubsection{Dimensión del desarrollo infantil}

Esta dimensión se aborda ampliamente en el currículo mediante diferentes asignaturas del área de especialización y psicopedagógicas, con un enfoque teórico esencialmente cognitivo, psicolingüístico y sociocultural. La bibliografía generalmente es actualizada y pertinente. A continuación se detallan los hallazgos de cada ámbito.

1) Teorías del desarrollo. Cinco de los ocho cursos que contemplan este ámbito lo relacionan explícitamente con el proceso de la LEI. En ellos se cubren las perspectivas teóricas, pero incluyen más detalles sobre las etapas de apropiación de la escritura que de la lectura.

2) Teorías del aprendizaje y la motivación. Es el ámbito más desarrollado del currículo prescrito, con doce asignaturas que lo abarcan. Resalta positivamente la adopción de la perspectiva crítico-reflexiva, que comprende el aprendizaje como proceso complejo y la escuela como institución social en la que los actores juegan un rol motivador.

3) Desarrollo del lenguaje. Se imparte en seis asignaturas del currículo. En ellas se trabajan temáticas que permiten al grupo de $\mathrm{DeF}$ analizar la alfabetización de la niñez en relación con el proceso evolutivo, y establecer sus implicaciones para las estrategias de enseńanza-aprendizaje. Sin embargo, no se profundiza en aquellas que permitan conocer las dificultades que se pueden enfrentar en cada una de las etapas de adquisición de la lengua escrita.

4) Fundamentos de la enseñanza-aprendizaje de la lectura. Las microhabilidades (decodificación, fluidez, vocabulario, construcción de oraciones y cohesión, conocimiento previo y razonamiento, memoria funcional y atención) y las estrategias de lectura para el primer ciclo son enunciadas y analizadas teóricamente en algunos casos. El estudio y práctica de situaciones didácticas exitosas no se acentúa.

5) Fundamentos de la enseńanza-aprendizaje de la escritura. La carrera aborda el tema de la escritura en toda su complejidad, desde las teorías psicolingüísticas referidas al proceso de alfabetización inicial hasta los enfoques didácticos para la enseñanza de la expresión escrita en sus niveles morfosintáctico, léxico, pragmático y discursivo.

6) Neurociencia de la LEI. Se abarca la neurociencia cognitiva y la reflexión sobre su importancia en el ámbito educativo, así como el proceso de adquisición y desarrollo del lenguaje oral y escrito. La propuesta enriquece la formación, pero quizá por la gran cantidad de contenidos incluidos, el abordaje llega a ser solamente teórico.

7) Psicología y sociología de la LEI. Los programas ofrecen un bagaje teórico amplio, pero no especifican su relación con la LEI.

8) Impacto del contexto en el aprendizaje de la LEI. Se incluyen los usos sociales de la lengua escrita, proporcionando al cuerpo de DeF herramientas para proponer una mediación ajustada a su forma de ver el mundo (Ferreiro \& Teberosky, 1979; Teberosky, 2000) y a sus tradiciones lingüísticas (Lucas \& Grinberg, 2008), tal como lo solicita el currículo dominicano vigente.

9) Trastornos del desarrollo de la LEI. El programa propone, desde una perspectiva cognitiva e interdisciplinaria, la reflexión sobre las difi- 
cultades más frecuentes en LEI y estrategias para trabajarlas, con una visión centrada en el desarrollo (Tunmer \& Hoover, 2019). No obstante, se necesita transitar de la teoría a la práctica.

\subsubsection{Dimensión del conocimiento de la LEI}

El programa de estudios aborda los once ámbitos de esta dimensión en diferentes cursos y con distintos grados de profundización.

1) Lenguaje oral. Resaltan entre los temas la oralidad como actividad lingüística, del discurso oral al escrito, los aspectos psicosociales de la actividad oral y los elementos verbales y paraverbales de la oralidad.

2) Concepto de lo impreso. No aparece explícitamente en el plan de estudio. Los contenidos y actividades del curso teórico brindan conocimientos básicos sobre cómo se trabajan los materiales impresos y las funciones que cumplen (Ferreiro, 1997).

3) Conciencia fonológica. Se aborda en una sola asignatura en la cual se aplica el método fónico para diferenciarlo de la conciencia fonológica y se subraya su importancia para el éxito del aprendizaje de la lectura, lo que coincide con diversas investigaciones (Sastre-Gómez et al., 2017).

4) Principio alfabético. Se proponen situaciones para favorecer la transición de las hipótesis de escritura de la niñez, considerando los indicadores básicos de alfabetización para el primer ciclo de primaria (Ferreiro, 2002) y el trabajo con estrategias cognitivas para promover la escritura a través de clases prácticas y demostraciones. No se establece explícitamente el modelado por parte del DF ni tampoco aparecen desarrollados otros componentes de este ámbito, de forma que sea objeto de una instrucción sistemática por parte de todo el profesorado.

5) Vocabulario. Se aborda en un curso diseñado para que el grupo de DeF se apropie de estra- tegias metodológicas que luego les permitirán intervenir en el aula real a través de actividades lúdicas.

6) Fluidez lectora. Se desarrolla explícitamente en una asignatura en la que se incluye la lectura en voz alta y, entre los criterios de evaluación, grabaciones de la lectura y una ficha de observación para determinar la fluidez, la entonación y el tono de voz.

7) Comprensión lectora. El programa de formación enfatiza la comprensión en todos sus niveles, aunque sin una vinculación explícita con el vocabulario y la fluidez lectora. Se reflexiona sobre diferentes teorías para facilitar al DeF el entendimiento del proceso, así como el conocimiento de las estrategias metodológicas que favorecen su adquisición.

8) Conexiones entre lectura y escritura. Este ámbito se aborda al comienzo de la carrera a través de la instrucción teórica de los siguientes temas: las relaciones entre el discurso oral y escrito, la oralidad como actividad lingüística y la escritura como código de transcripción versus la escritura como sistema de representación.

9) Grafomotricidad. Se aborda en una asignatura que presenta al DeF las etapas del desarrollo del grafismo - precaligráfica, caligráfica infantil y poscaligráfica - y las microhabilidades de la expresión escrita.

10) Expresión escrita. Junto con la comprensión lectora, es uno de los ámbitos de más amplia cobertura en la formación. Se incluyen los procesos cognitivos interactivos y recursivos en la producción de textos propuestos por el currículo vigente. Sin embargo, el porcentaje práctico y de modelado de estrategias del DF es menor que el estipulado.

11) Convenciones de la escritura. Se aborda en cuatro asignaturas que comprenden la teoría y práctica de la morfología y la sintaxis, considerando al texto como unidad. También se 
contemplan los aspectos formales del grafismo y las etapas del proceso de adquisición de la lengua escrita, así como las microhabilidades de la expresión escrita.

\subsubsection{Dimensión de la evaluación}

El desarrollo pormenorizado de los seis ámbitos de esta dimensión fue identificado en cinco asignaturas, en las que se analizan los aportes teóricos y metodológicos sobre la evaluación de la LEI, tomando en cuenta los indicadores de logro del primer ciclo de primaria.

Para los ámbitos propósitos de la evaluación, tipos de evaluación y evaluación de los aprendizajes, se proveen los fundamentos teóricos y metodológicos de la evaluación educativa para la toma de decisiones orientada a la mejora continua de la calidad del proceso de enseńanza-aprendizaje, respetando la dignidad y las diferencias individuales del estudiantado.

En el plan de estudios, para el diseño y aplicación de herramientas de evaluación e interpretación y uso de resultados, se detallan técnicas, instrumentos y criterios de evaluación. En las propuestas didácticas prescritas para el grupo de DeF, no siempre se detalla el tipo de evaluación propuesta (formativa o sumativa).

\subsection{Pregunta 2}

Se identificaron numerosas fortalezas en las categorías generales analizadas, tales como un programa de formación centrado en el primer ciclo, constructivista, con especialización en LEI y coherente con el currículo de los primeros grados del MINERD. También se aprecian algunas brechas sobre las que es necesario reflexionar.

En lo que respecta a la comprensión de la LEI, se percibieron diferencias entre las sedes, pues algunos DF explicaron que no fueron formados específicamente en la alfabetización inicial y, por lo tanto, ponen en práctica un enfoque más centrado en la gramática que en los procesos cognitivos y los textos, como lo estipula la evidencia consultada y el currículo vigente.

Un gran propósito y fortaleza institucional del programa de formación es la experiencia práctica en las escue- las, organizada desde una coordinación general, que garantiza el seguimiento a profesores acompañantes y a docentes en servicio que reciben al grupo de $\mathrm{DeF}$ en sus aulas. A pesar de esto, algunos DeF no se sienten tan seguros de sus conocimientos en LEI al final de la carrera como para enseñarla, lo que puede ser causado por el mayor peso de la teoría sobre la práctica.

Otra divergencia entre la propuesta curricular y la percepción de DeF es la relacionada con la evaluación del aprendizaje de niñas y niños. Aunque se destaca por sus fortalezas en los programas, al preguntar a DeF manifestaron que no dominan esta dimensión. Se sugiere enfatizar su modelado en los distintos temas relacionados con la LEI.

En cuanto a la evaluación de los aprendizajes del grupo de DeF, se encontró que se privilegia saber cómo aplican conocimientos, estrategias y actitudes en la resolución de problemas reales o simulados relacionados con la LEI mediante diferentes tipos de evaluación, con el propósito de identificar fortalezas y debilidades para superarlas. Debe realizarse un esfuerzo adicional para que se apropien de criterios relacionados con la LEI que les preparen para hacer evaluaciones de manera autónoma y sustentada.

Sobre el diseño por competencias de los programas también se encontró otra brecha. Se identificó cierta desarticulación entre las competencias enunciadas (fundamentales, genéricas y específicas) y los contenidos, las estrategias, las actividades formativas y las técnicas y criterios de evaluación. Así, por ejemplo, los contenidos aparecen en forma de lista de temas en los programas, sin clasificación por unidades ni articulación directa con las competencias planteadas. Los syllabus, en cambio, se organizan por unidades temáticas, pero en ellas no se mencionan las competencias.

Respecto a la bibliografía, si bien existe una lista de referencias en cada asignatura, se observaron diferencias entre los programas y los syllabus. En las entrevistas a DF, se constató que se deben a que los programas fueron propuestos centralmente por la institución y los syllabus por el cuerpo docente. 


\subsection{Pregunta 3}

\subsubsection{Dimensión 1. Desarrollo infantil}

La carrera prescribe reflexión y práctica de las dificultades lectoras y escriturales más frecuentes en el primer ciclo de primaria desde una visión centrada en el desarrollo, lo que coincide con la perspectiva de DF entrevistados.

En las aulas se observaron clases con dinámicas de participación activa y con uso de recursos variados sobre los factores biológicos, físicos y sociales para el desarrollo del lenguaje, así como sobre las teorías de adquisición de la lengua. Sin embargo, el grupo de DeF no coincidió en sus apreciaciones respecto a la puesta en práctica de esta dimensión: "Yo considero sinceramente, que nos han preparado con métodos teóricos, pero nos faltan las pistas de cómo poder aplicarlos con los niños". (Código: Categorías generales $\backslash 2$. Prácticas pedagógicas Peso: 0 Entrevistas DeflDeF 9 Posición: 11 - 11)

\subsubsection{Dimensión 2. Conocimiento de la LEI}

La importancia dada a la comprensión lectora y a la expresión escrita, así como a las convenciones de la escritura, se destacan como una fortaleza para esta dimensión, tanto en el currículo prescrito como en el aplicado. En efecto, en el plan de estudio, estos ámbitos son desarrollados en varias asignaturas y en las entrevistas a DF todos coincidieron en que son centrales para el desarrollo de la LEI. También el grupo de DeF dio peso a estos tres ámbitos y expresó gusto por su puesta en práctica en las aulas.

Los ámbitos del vocabulario, la conciencia fonológica, el principio alfabético (la decodificación), el concepto de lo impreso y la fluidez lectora están escasamente detallados en el currículo prescrito. Sin embargo, en el aplicado se observó en dos clases la práctica de la conciencia fonológica. Este hecho se vio corroborado en una entrevista a la DF.

Igualmente se observó en otra clase el trabajo con el ámbito principio alfabético. Cada una de las habilidades fue modelada y practicada. La DF facilitó el trabajo a partir de una receta y las DeF usaron diversos mate- riales manipulativos (tapitas para decodificar las sílabas de cada palabra, bingos y crucigramas, entre otros).

En el ámbito concepto de lo impreso, también se registraron diferencias entre el currículo prescrito y el aplicado. En efecto, en el prescrito figura explícitamente en una asignatura y con un enfoque más teórico que práctico, mientras que en el currículo aplicado se incluyó en otra asignatura, como pudo observarse en el aula, con modelación del DF y práctica a partir de un libro de cuentos de una DeF. Esta situación no se percibió de la misma manera en todos los recintos ni entre todas las personas entrevistadas, lo que hace pensar que la enseñanza no está estandarizada y que puede haber vacíos, por la falta de especificación en el currículo prescrito, en otros salones que no se observaron.

En cuanto al vocabulario, en las clases observadas se constató la presencia circunstancial de este ámbito, cuando DeF modelaban la lectura de cuentos o sus DF solicitaban sinónimos. El cuerpo de DF destacó su importancia, pero ninguno en el grupo de DeF entrevistados lo citó como una habilidad de la LEI. Por otra parte, algunos componentes de la fluidez lectora (entonación, precisión, tono de la voz) fueron resaltados en algunas entrevistas y practicados en clase por DF, pero el grupo de DeF no los mencionó.

\subsubsection{Dimensión 3. Evaluación}

En las entrevistas a DF y a DeF, se constataron diferencias entre lo prescrito y lo aplicado respecto a esta dimensión. Así, una DF expresó que sería conveniente que el cuerpo docente que imparte evaluación sea del área de especialización para que se ponga en práctica lo que el plan de estudio prescribe respecto a la LEI. Se encontraron igualmente vacíos formativos cuando los DeF afirman que no se sienten cómodos con el manejo de los temas de evaluación, aunque se los hayan enseñado, o cuando sostienen que han aprendido a diseñar las evaluaciones, pero solo las han aplicado entre el grupo de estudiantes.

\subsubsection{Categorias generales}

Respecto al enfoque teórico de la enseñanza de la LEI, se detectaron diferencias entre el currículo prescrito y 
el aplicado. En las entrevistas a DeF, se manifestaron diversos énfasis teóricos sobre conocimientos de la LEI según los recintos en los que estudian, tal como puede observarse en estos ejemplos contrapuestos: "La motricidad es una de las habilidades básicas de LEI, la cual se puede desarrollar permitiendo a los niños dibujar y que tracen líneas. A su vez, podemos enseñarles a través de la repetición y las caligrafías". (Código: Categorías\D2 \9. Grafomotricidad Peso: 0 Entrevistas DeflDeF12 Posición: 9-9)

Esta entrevista revela apego a un enfoque mecanicista de la enseñanza de la LEI, a diferencia de la siguiente en la que se observa que se comprende la importancia de la relación entre lengua oral y escrita:

Hay muchas metodologías buenas que nos muestran en la carrera para enseñar la LEI; está el método clásico, el cual es el alfabético, donde el docente por medio del alfabeto enseńa las vocales y las letras a los niños, y el método fonético, en el cual el estudiante va aprendiendo por medio de lo que escucha (Código: Categorías $\backslash D 2 \backslash 2$. Conocimiento fonológico Peso:0 Entrevistas DefDeF10 Posición: 9 - 9)

Finalmente, en el siguiente ejemplo puede percibirse que se conoce cuáles son las competencias que se deben tener en cuenta al enseñar la LEI: "Entre las habilidades básicas que los niños deben desarrollar están la comprensión oral y escrita y las competencias de producción escrita”. (Código: Categorías\D2\10. Expresión escrita Peso: 0 Entrevistas DeflDeF6 Posición: 9-9)

En cuanto a la aplicación del enfoque de enseñanza, aunque en las observaciones de aula se pudo percibir el esfuerzo de DF por colocar al estudiantado como protagonista de sus procesos de enseñanza-aprendizaje, en algunas prácticas pedagógicas se percibieron estilos que atentan contra este enfoque, como, por ejemplo, clases muy teóricas centradas en la exposición de la profesora del curso, con escasa participación y motivación estudiantil.

Sobre la bibliografía, en las entrevistas a DF se refleja conocimiento de las referencias que figuran en el currículo prescrito; en cambio, el grupo de DeF no las consulta directamente, lo que provoca un manejo fragmentado de la información de la carrera.

Con relación al tiempo previsto y al tiempo real dedicado a la enseńanza de la LEI, una gran parte de las asignaturas tiene tres créditos, dos prácticos y uno teórico. En las clases observadas, la mayoría de DF respetó el tiempo prescrito. Sin embargo, no siempre el tiempo dedicado a la práctica se correspondió con los créditos estipulados.

Finalmente, el currículo prescrito enfatiza la cultura lectora, contemplando incluso un club de lectura en sus diferentes sedes, abierto a todo el estudiantado. El grupo de DeF manifestó gusto por la lectura por placer, aunque se citó el tiempo como una limitación para lograrlo. Sin embargo, una DF dijo que muy pocos estudiantes de la licenciatura en Educación Primaria se motivan por asistir al club de lectura, pues, de todos los participantes, solo dos son de esa concentración.

\section{Discusión y conclusiones}

Los resultados de esta investigación constituyen un insumo para la reflexión y mejora de la lectoescritura en el plan de estudios de la licenciatura en Educación Primaria Primer Ciclo. Se espera que sus hallazgos fortalezcan aún más un programa que ya tiene múltiples aspectos positivos. Asimismo, es deseable que este estudio sea tomado en cuenta en la toma de decisiones de las políticas curriculares y de formación continua del cuerpo docente a nivel nacional.

Respecto a la primera pregunta del estudio: ¿cómo se alinea el currículo de formación inicial docente con la evidencia sobre cómo se aprende la lectoescritura en los primeros ańos y qué debe saber y poder hacer el cuerpo docente para una instrucción efectiva?, los resultados de la investigación realizada en la República Dominicana indican lo siguiente: Aunque el plan de estudios de la carrera de Educación Primaria Primer Ciclo se encuentra alineado con la evidencia estudiada, pues aborda la mayor parte de los ámbitos de las dimensiones necesarias para enseñar y aprender la LEI, no todos esos ámbitos aparecen 
con el peso requerido por la investigación científica (vocabulario, conciencia fonológica). Esto podría repercutir en el desarrollo de los procesos cognitivos y metacognitivos que intervienen en la comprensión lectora y en la producción escrita, lo que amerita una revisión.

Por otra parte, algunos ámbitos no están suficientemente detallados (concepto de lo impreso, principio alfabético, fluidez lectora), y su puesta en práctica no siempre reveló el uso de metodologías de éxito en pos de un desarrollo integral del proceso de lectura y escritura. Al explicitarlos más pormenorizadamente en el programa propuesto e incluirlos en actividades que otorguen sentido a las prácticas de lectura y escritura, el grupo de DeF contaría con más herramientas para poder intervenir con eficacia en las aulas de los primeros grados.

Se necesita, por lo tanto, un mayor esfuerzo para tender puentes entre teoría y práctica, tanto en la formulación de los programas del plan de estudio, en las actuaciones de DF y DeF, como en las experiencias de campo. También es necesario integrar las competencias del saber con las del saber hacer. Así, el grupo de DeF podría aplicar los conocimientos adquiridos en beneficio de mejores logros en LEI por parte de la niñez, tal como las premisas teóricas de esta investigación lo demuestran, y así superar cierta tensión e insatisfacción que esta ausencia les genera.

Con relación a la segunda pregunta: ¿cuáles son las fortalezas y las brechas del plan de estudios de formación inicial docente en relación con la evidencia investigativa?, se encontraron más fortalezas que brechas. Entre las primeras: contar con un programa coherente con el currículo dominicano vigente, con una estructura curricular determinada por el MESCyT (común a todas las instituciones de educación superior) y especializado en LEI en los primeros grados, lo que permite asegurar una preparación como especialistas de DeF que podrán contribuir con una mayor calidad en la enseñanza de la LEI.

Sin embargo, esta investigación presenta una oportunidad para reflexionar y trazar planes para superar las brechas. Se pudo constatar diferencias entre los tres recintos en los que se realizó la investigación que atañen esencialmente al enfoque teórico, a las metodologías y prácticas pedagógicas que asumen, así como al tipo de evaluación que enfatizan, situación que se debe principalmente a que no todo el cuerpo docente se ha formado en lectoescritura, a pesar de ostentar titulación de maestrías y los perfiles laborales y personales requeridos.

Así, por ejemplo, algunos DF tienen un enfoque más centrado en los procesos gramaticales de la lengua, alejándose de la propuesta comunicativa y cognitiva del currículo dominicano vigente y de lo que la evidencia consultada sugiere. Otro aspecto a sopesar es el modelado por parte de los formadores, pues si bien está presente en las observaciones de clases efectuadas, se hace necesario sistematizar esta práctica para que todo el equipo docente la aplique. Igualmente se debe prestar atención a la percepción del grupo de DeF, pues varios manifestaron que no dominan el ámbito de la evaluación de los niños y niñas, lo que marca una diferencia con la propuesta del plan de estudio, que presenta fortalezas en esta dimensión por su orientación procesual.

Por otro lado, es necesaria una formación continua de quienes ejercen como DF para que puedan aplicar el currículo de manera coherente y significativa, y garantizar que el grupo de DeF llegue al final de su carrera con competencias de calidad respecto a cada uno de los ámbitos de la LEI. De esta forma podrá contribuir con mejores logros de la niñez de la República Dominicana en la decodificación y en la comprensión literal, inferencial y crítica, para superar los magros resultados obtenidos hasta ahora en tercer grado en las evaluaciones de la UNESCO y en las evaluaciones diagnósticas nacionales.

Sobre la tercera pregunta: ¡en qué se diferencia el currículo prescrito del currículo realmente aplicado en los programas de formación inicial docente en el ámbito de la LEI?, los resultados reflejaron, en primer lugar, la importancia dada a la comprensión lectora, la expresión escrita y las convenciones de la escritura tanto en el currículo prescrito como en el aplicado, lo que va de acuerdo con el carác- 
ter central de estos ámbitos en las investigaciones consultadas. Sin embargo, otros ámbitos de la LEI (vocabulario, conciencia fonológica, principio alfabético, concepto de lo impreso y fluidez lectora) no tuvieron tanta presencia en el plan de estudio, aunque pudo observarse que algunos DF los incluyeron en sus clases. Pero como los programas deben ser la guía para la ejecución de un currículo y no a la inversa, es necesario ampliar la presencia de estos ámbitos en el plan de estudio para evitar diferencias entre los recintos y entre los formadores, con el correspondiente impacto en los logros del estudiantado del primer ciclo de primaria.

En segundo lugar, la práctica de los contenidos en los cursos necesita fortalecerse, disminuyendo las exposiciones de DeF y acentuando metodologías y estrategias para abordar cada contenido del ámbito de la LEI de manera sistemática. Con ello se lograría mejorar la actuación del futuro cuerpo de docentes en las Prácticas I a VI en las aulas reales y disminuiría la necesidad de talleres adicionales para suplir las ausencias detectadas. En este sentido, el modelado de parte de quienes ejecutan la formación debe quedar explícito en los programas de cada asignatura para guiar al cuerpo de profesores de las diferentes sedes a ponerlo en práctica en sus asignaturas. Cabe señalar que la mayoría de los cursos se encuentran estructurados en tres horas presenciales, una teórica y dos horas prácticas; sin embargo, esta disposición no siempre se cumple.

En tercer lugar, se propone afianzar aún más la cultura lectora del grupo de futuros maestros. En lo que atañe a su formación, muchos estudiantes cursan y aprueban los cursos sin haber usado las fuentes bibliográficas de los programas, estudiando básicamente de sus notas o presentaciones de clase, situación que coincide con hallazgos de otros estudios (Cartolari \& Carlino, 2012). Por otra parte, no todo el grupo ama la lectura por placer, aspecto a superar, pues diferentes investigaciones (Applegate A. \& Applegate M., 2004; Nathanson et al., 2008) han demostrado una relación significativa entre la cantidad de libros leídos por estudiante y el haber contado en la escuela con docentes entusiasmados por la lectura.

\subsection{Limitaciones}

Esta investigación tuvo limitaciones que deben ser consideradas para entender su alcance. Solamente contempló el programa de formación inicial de docentes de una institución superior de la República Dominicana que, aunque se ajusta a la estructura curricular propuesta por el MESCyT (2015), tiene sus particularidades. Por esta razón, y porque otras universidades ofrecen esta licenciatura, no es posible generalizar los hallazgos.

Por otro lado, el tiempo y cronograma de la investigación - llevada a cabo en dos períodos del año académico- limitaron el trabajo de observación y de entrevistas a DF y DeF a las clases que se ofrecían en dichos períodos. Igualmente, la distancia geográfica entre los recintos y la sede de la investigación (Santiago), así como los horarios en los que se impartían las asignaturas especializadas, limitaron la observación de clases.

\subsection{Recomendaciones}

A continuación se detallan las recomendaciones sugeridas para cada una de las tres dimensiones del estudio.

\subsubsection{Dimensión 1: Desarrollo infantil}

Será importante balancear el peso de algunos ámbitos, incluir práctica en varios en los que actualmente hay vacíos, acordar igual énfasis a las microhabilidades de lectura que a las de escritura, e incorporar teoría y práctica sobre la transferencia de habilidades de otra lengua materna al espańol.

\subsubsection{Dimensión 2: Conocimiento de la LEI}

Explicitar en el plan de estudio la importancia del modelado, espacios de práctica, y uniformar la aplicación del currículo en todos los recintos. Dar mayor peso a algunos ámbitos de la dimensión escasamente enseñados, asignar profesores especializados en alfabetización inicial a los cursos de LEI y afianzar en el estudiantado el manejo directo de las fuentes bibliográficas. 


\subsubsection{Dimensión 3: Evaluación}

Es necesario enfatizar el modelado de la evaluación de cada ámbito de la LEI en las diferentes asignaturas de especialización e incluir mayor cantidad de prácticas reales con estudiantes. Incluir reflexión y práctica sobre las evaluaciones diagnósticas de comprensión lectora y sobre las de la UNESCO.

\section{Agradecimientos}

A la Agencia de los Estados Unidos para el Desarrollo Internacional (USAID), por su apoyo a la Red para la Lectoescritura Inicial de Centroamérica y del Caribe (RedLEI), liderada por la Universidad del Valle de Guatemala, al American Institutes for Research y a la Pontificia Universidad Católica Madre y Maestra, a través de su Centro de Excelencia para la Investigación y Difusión de la Lectura y la Escritura (CEDILE)..

\section{Referencias bibliográficas}

Adger, C. T., Snow, C. E., \& Christian, D. (Eds.). (2018). What teachers need to know about language. Pennsylvania: Multilingual Matters.

Alamargot, D., \& Chanquoy, L. (2001). Through the models of writing. Dordrecht: Springer.

Alviz, G. (2012). La grafomotricidad en educación infantil. Revista Arista Digital, 16(6), 48-54.

http://r.issu.edu.do/l.php?l=130RCR

American Speech-Language-Hearing Association (ASHA). (2008). Roles and Responsibilities of Speech-Language Pathologists in Early Intervention: Guidelines. http://r.issu.edu.do/l.php?l=1294Je

Andrade-Calderón, P., Stone, R., \& Vijil, J. (2020) ¿Cómo se está formando al cuerpo docente centroamericano para enseńar la lectoescritura inicial? Aportes de una investigación regional. Actualidades Investigativas en Educación, 20(2), 1-33.

https://doi.org/10.15517/aie.v20i2.41588

Applegate, A., \& Applegate, M. (2004). The Peter Effect: Reading habits and attitudes of preservice teachers. The Reading Teacher, 57(6), 554-563.
Arancibia, B., Bizama, M., \& Sáez, K. (2012). Aplicación de un programa de estimulación de la conciencia fonológica en preescolares de nivel transición 2 y alumnos de primer año básico pertenecientes a escuelas vulnerables de la Provincia de Concepción, Chile. Revista Signos, 45(80), 236-256. https://doi.org/10.4067/S0718-09342012000300001

Asociación Americana de Psiquiatría. (2013). Guia de consulta de los criterios diagnósticos del DSM 5. Arlington: Asociación Americana de Psiquiatría.

August, D., \& Shanahan, T. (Eds.). (2006). Developing literacy in second-language learners: Report of the National Literacy Panel on Language-Minority Children and Youth. Mahwah, NJ: Lawrence Erlbaum.

Avendaño, F., \& Miretti, M. (2006). El desarrollo de la lengua oral en el aula. Rosario: Homo Sapiens Ediciones.

Barletta-Manjarrés, N., Cortez-Román, N. A., \& Medzerian, S. (2012). From official educational policy to the composition classroom: Reproduction through metaphor and metonymy. Journal of Writing Research, 4(1), 31-51.

https://doi.org/10.17239/jowr-2012.04 01.2

Bazerman, C., Farmer, F., Halasek, K., \& Williams, J. M. (2005). Responses to Bakhtin's “Dialogic Origins and Dialogic Pedagogy of Grammar: Stylistics as Part of Russian Language Instruction in Secondary Schools": Further Responses and a Tentative Conclusion. Written Communication, 22(3), 363-374. https://doi.org/10.1177/0741088305278032

Bellei, C., Poblete, X., Sepúlveda, P. , Orellana, V., \& Abarca, G. (2013). Situación Educativa de América Latina y el Caribe: Hacia la educación de calidad para todos al 2015. Santiago: Oficina Regional de Educación para América Latina y el Caribe (ORELAC/ UNESCO Santiago).

Berman, R. A. (2004). Between emergence and mastery: The long developmental route of language acquisition. En R.A. Berman (Ed.). Language development across childhood and adolescence (pp. 9-34). Amsterdam: John Benjamins. 


\section{MONTENEGRO-DE-OLLOQUI}

Fortalezas y áreas de mejora de la formación inicial docente en lectoescritura...

Berninger, V. W., \& Amtmann, D. (2003). Preventing written expression disabilities through early and continuing assessment and intervention for handwriting and/or spelling problems; Research into practice. En H. L. Swanson, K. R. Harris, \& S. Graham (Eds.), Handbook of learning disabilities (pp. 345-363). New York: Guilford Press.

Bezemer, J., \& Kress, G. (2010). Changing Text: A Social Semiotic Analysis of Textbooks. Designs for Learning, 3(1-2), 10.

https://doi.org/10.16993/dfl.26

Bialystok, E. (2011). Reshaping the mind: the benefits of bilingualism. Canadian Journal of Experimental Psychology/Revue canadienne de psychologie expérimentale, 65(4), 229-235.

https://doi.org/10.1037/a0025406

Bravo, L., Villalón, M., \& Orellana, E. (2011). La conciencia fonológica y la lectura Inicial en niños que Ingresan a primer año básico. Psykhe, 11(1), 175-182. http://r.issu.edu.do/l.php?l=131aVt

Brem, S., Bach, S., Kucian, K., Kujala, J. V., Guttorm, T. K., Martin, E., \& Richardson, U. (2010). Brain sensitivity to print emerges when children learn letter-speech sound correspondences. Proceedings of the National Academy of Sciences, 107(17), 7939-7944.

Bruning, R., \& Kauffman, D. (2015). Self-efficacy beliefs and motivation in writing development. En C. A. MacArthur, S. Graham, \& J. Fitzgerald (Eds.). Handbook of Writing Research (2nd ed.). (pp. 160173). Nueva York: Guilford Press.

Bruns, B., \& Luque J. (2015). Profesores Excelentes: Cómo mejorar el aprendizaje en América Latina y el Caribe. Washington DC: Grupo del Banco Mundial.

Bui, Y. N., Schumaker, J. B., \& Deshler, D. D. (2006). The effects of a strategic writing program. Disabilities Research and Practice, 2, 244-260.

https://doi.org/10.1111/j.1540-5826.2006.00221.x

Camargo, G., Montenegro, R., Maldonado, S., \& Magzul, J. (2013). Aprendizaje de la lectoescritura. Guatemala: USAID/Reforma Educativa en el Aula.
Cartolari, M., \& Carlino, P. (2012). Leer y escribir en la formación docente: aportes de las investigaciones anglosajonas. Acción pedagógica, 21(1), 6-17. http://r.issu.edu.do/l.php?l=132Sbq

Casey, B., Davidson, M., \& Brice, R. (2002). Functional magnetic resonance imaging: basic principles of and application to developmental science. Developmental Science, 5(3), 301-309. https://doi.org/10.1111/1467-7687.00370

Casado, M. (2000). Lingüística y gramática del texto: su articulación interdisciplinar. Revista de Filología Hispánica, 16(2), 247-262.

https://revistas.unav.edu/index.php/rilce/article/view/26801

Cassany, D. (2012). Describir el escribir. Cómo se aprende a escribir. Barcelona: Paidós.

Chappuis, S., \& Chappuis, J. (2008). The Best Value in Formative Assessment. Educational Leadership, 65(4), 14-19.

http://r.issu.edu.do/l.php?l=133mmW

Chesterfield, R., \& Abreu-Combs, A. (2011). Center for excellence in teacher training (CETT) two-year impact study report (2008-2010). Washington D.C: USAID Bureau for Latin America and Caribbean.

Condemarín, M., \& Medina, A. (2000). El diario en el aula. Santiago: LOM.

Copple, C., \& Bredekamp, S. (2009). Developmentally appropriate practice in early childhood programs serving children from birth through age 8. Washington, DC: National Association for the Education of Young Children (NAEYC).

Darling-Hammond, L., \& Bransford, J. (2005). Preparing teachers for a changing world: What teachers should learn and be able to do. San Francisco: Jossey-Bass.

Defior, S., \& Serrano, F. (2011). La conciencia fonémica, aliada de la adquisición del lenguaje escrito. Revista de Logopedia, Foniatría y Audiología, 31(1), 2-13. https://doi.org/10.1016/S0214-4603(11)70165-6

De Mier, M., Borzone, A., \& Cupani, M. (2012). La fluidez lectora en los primeros grados: relación entre 
habilidades de decodificación, características textuales y comprensión. Un estudio piloto con niños hablantes de español. Revista Neuropsicología Latinoamericana, 4(1), 18-33.

Díaz, C., Villalon, M., \& Adlerstein, C. (2015). Conocimiento práctico para la enseñanza del lenguaje oral y escrito de estudiantes de educación inicial: Una aproximación desde la elaboración de mapas conceptuales. Interdisciplinaria, 32(2), 331-345. http://r.issu.edu.do/l.php?l=134NPc

Duque, P. , Rodríguez, J., \& Vallejo, S. (2013). Prácticas Pedagógicas y su relación con el Desempeño Académico. Colombia: Universidad de Manizales.

Faigley, L., \& Witte, S. P. (1981). Coherence, cohesion, and writing quality. College Composition and Communication, 32(2), 189-204.

https://doi.org/10.2307/356693

Ferreiro, E. (1997). Alfabetización. Teoría y práctica. México: Siglo XXI.

Ferreiro, E. (2002). Relaciones de (in)dependencia entre oralidad y escritura. Barcelona: Gedisa.

Ferreiro, E., \& Gómez, M. (1986). Nuevas perspectivas sobre los procesos de lectura y escritura. Buenos Aires: Siglo XXI.

Ferreiro, E., \& Teberosky, A. (1979). Los sistemas de escritura en el desarrollo del niño. México: Siglo XXI editores.

Ferreiro, E., Teberosky, A., Castorina J., Grunfeld D., Avendaño A., \& Báez, M. (2002). Sistema de escritura, constructivismo y educación. A veinte años de la publicación de los sistemas de escritura en el desarrollo del niño. Rosario: Homo Sapiens Ediciones.

Fons, M. (2004). Leer y escribir para vivir. Alfabetización inicial y uso real de la lengua escrita en la escuela. Barcelona: Graó.

Gallego, J. L., García, A., \& Rodríguez, A. (2014). Estrategias metacognitivas en la expresión escrita: un estudio de caso con futuros docentes. Bordón, 66(4), 39-53.

https://doi.org/10.13042/Bordon.2014.66404
Genesee, F., Lindholm-Leary, K., Saunders, W., \& Christian, D. (2006). Educating English language learners. New York: Cambridge University Press.

Goldman-Rakic, P. S. (1987). Development of Cortical Circuitry and Cognitive Function.

Child Development, 58(3), 601-622.

http://doi.org/10.2307/1130201

Graham, S., \& Hebert, M. A. (2010). Writing to read: Evidence for how writing can improve reading. A Carnegie Corporation Time to Act Report. Washington, DC: Alliance for Excellent Education.

Hayes, J. R. (1996). A new framework for understanding cognition and affect in writing. En C. M. Levy \& S. Ransdell (Eds.), The science of writing: Theories, methods, individual differences and applications (pp. 1-27). Mahwah, NJ: Lawrence Erlbaum Associates.

Hayes, J. R., \& Flower, L. S. (1980). Identifying the organization of writing processes. En L. Gregg \& E. R. Steinberg (Eds.), Cognitive processes in writing (pp. 3-30). Hillsdale, NJ: Lawrence Erlbaum Associates.

Holdgreve-Resendez, R. R. (2010). Concepts of Print (cont.). Extracted from Concepts of Print and Genre Slides from angel.msu.edu on September 22, 2010. TE 301: Learners and Learning in Context.

https://megosliteraryreferenceproject.wordpress. com/2010/12/01/concepts-of-print

Hoover, W. A., \& Gough, P. B. (1990). The simple view of reading. Reading and Writing: An Interdisciplinary Journal, 2(2), 127-160.

https://doi.org/10.1007/BF00401799

Hoyle, S., \& Adger, C. T. (1998). Introduction. En S. M. Hoyle \& C. T. Adger (Eds.). Kids Talk: Strategic Language Use in Later Childhood (pp. 3-22). New York: Oxford University Press.

International Literacy Association (2018). Standards for the preparation of literacy professionals 2017. Newark, DE: International Literacy Association.

Kaufman, A. M., Lerner, D., \& Castedo, M. (2015). Leer y aprender a leer: Documento transversal 2. Alfabetización en la Unidad Pedagógica. Especialización 
Docente de Nivel Superior. Buenos Aires: Ministerio de Educación de la Nación.

Leppänen, P. H. T., Hämäläinen, J. A., Salminen, H. K., Eklund, K. M., Guttorm, T. K., Lohvansuu, K., Lyytinen, H., \& Puolakanaho, A. (2010). Newborn brain event-related potentials revealing atypical processing of sound frequency and the subsequent association with later literacy skills in children with familial dyslexia. Cortex 46(10), 1362-1376. https://doi.org/10.1016/j.cortex.2010.06.003

Liston, C., Watts, R., Tottenham, N., Davidson, M. C., Niogi, S., Ulug, A. M., \& Casey, B. J. (2005). Frontostriatal microstructure modulates efficient recruitment of cognitive control. Cerebral Cortex, 16(4), 553-560.

https://doi.org/10.1093/cercor/bhj003

Litt, D. G., Martin, S. D., \& Place N. A. (2015). Literacy Teacher Education. Principles and Effective Practices. New York: The Guilford Press.

Lucas, T., \& Grinberg, J. (2008). Responding to the linguistic reality of mainstream classrooms: Preparing all teachers to teach English language learners. En M. Cochran-Smith, S. Feiman-Nemser \& J. McIntyre (Eds.). Handbook of research on teacher education: Enduring issues in changing contexts (pp. 606- 636). Nueva Jersey: Lawrence Erlbaum.

Manghi, D. (2011). La perspectiva multimodal sobre la comunicación. Desafíos y aportes para la enseñanza en el aula. Diálogos Educativos, 21(22), 4-15. http://r.issu.edu.do/l.php?l=13543X

Martínez, J., \& Montenegro, L. (2010). Plan Operativo de Capacitación Docente, Planes Operativos de Capacitación (pp. 139-164). México: CETT-CARD / ILCE.

McEwan, P. J. (2015). Improving learning in primary schools of developing countries: A meta-analysis of randomized experiments. Review of Educational Research, 85(3), 353-394.

https://doi.org/10.3102/0034654314553127
McField, G. P., \& McField, D. R. (2014). The consistent outcome of bilingual education programs: A meta-analysis of meta-analyses. En G. P. McField (Ed.). The miseducation of English learners (pp. $267-$ 298). Charlotte, NC: Information Age.

McKenna, M., \& Stahl, K. (2009). Assessment for reading instruction. New York: The Guilford Press.

McLaren, D. G., Ries, M. L., Xu, G., \& Johnson, S. C. (2012). A generalized form of context-dependent psychophysiological interactions (gPPI): A comparison to standard approaches. NeuroImage, 61(4), 1277-1286.

https://doi.org/10.1016/j.neuroimage.2012.03.068

Menyuk, P., \& Brisk, M. (2005). Language Development and Education: Children with Varying Language Experiences. London: Palgrave Macmillan UK.

Ministerio de Educación de la República Dominicana (MINERD). (2016). Diseño curricular nivel primario primer ciclo (1ro., 2do. y 3ro.). Santo Domingo: MINERD.

Ministerio de Educación de la República Dominicana (MINERD). (2017). Resultados de la evaluación diagnóstica nacional de tercer grado de primaria. Informe nacional. Santo Domingo: Dirección de la Evaluación de la Calidad.

Ministerio de Educación Superior, Ciencia y Tecnología (MESCyT). (2015). Normativa para la Formación Docente de Calidad en la República Dominicana. Santo Domingo: MESCyT.

Moats, L. (2009). Knowledge foundations for teaching reading and spelling. Reading and Writing, 22(4), 379-399.

https://doi.org/10.1007/s11145-009-9162-1

Nathanson, S., Pruslow, J., \& Levitt, R. (2008). The Reading Habits and Literacy Attitudes of Inservice and Prospective Teachers: Results of a Questionnaire Survey. Journal of Teacher Education, 59(4), 313321. https://doi.org/10.1177/0022487108321685

National Institute of Child Health and Human Development. (2000). Report of the National Reading 
Panel. Teaching children to read: An evidence-based assessment of the scientific research literature on reading and its implications for reading instruction: Reports of the subgroups (NIH Publication No. 00-4754). Washington, DC: U.S. Government Printing Office. http://www.nichd.nih.gov/ publications/nrp/report.htm

National Reading Panel (U.S.). (2000). Teaching Children to Read: An Evidence-Based Assesment of the Scientific Research Literature on Reading and Its Implications for Reading Instruction. Reports of the Subgroups. NIH Publication No. 00-4754. http://r.issu. edu.do/l.php?1=136kiz

National Research Council. (2010). Preparing Teachers: Building Evidence for Sound Policy. Washington, D.C.: The National Academies Press.

Olesen, P. , Westerberg, H., \& Klingberg, T. (2004). Increased prefrontal and parietal activity after training of working memory. Nature Neuroscience, 7(1), 75. https://doi.org/10.1038/nn1165

Ortega, L. (2008). Understanding second language acquisition. Londres: Hodder Education.

Patiño, G. (2005). Citas y referencias bibliográficas. Bogotá: Pontificia Universidad Javeriana.

Perrenoud, P. (2008). La evaluación de los alumnos. De la reproducción de la excelencia a la regulación de los aprendizajes. Entre dos lógicas. Buenos Aires: Ediciones Colihue.

Regalado, O., Tantaleán, L., \& Vargas, M. (2016). El Monitoreo pedagógico en el desempeño profesional docente. Perú: Didáctica, Innovación y Multimedia (DIM).

Rivkin, S. G., Hanushek, E. A., \& Kain, J. F. (2005). Teachers, schools and academic achievement. Econometrica, 73(2), 417-458.

Rolstad, K., Mahoney, K., \& Glass, G. (2005). The big picture: a meta-analysis of program effectiveness research on English Language Learners. Educational Policy, 19(4), 572-594.

https://doi.org/10.1177/0895904805278067
Romaine, S. (1995). Bilingualism. Oxford UK: Blackwell Publishers Inc.

Sáez Vega, R. (2015). De las destrezas aisladas al lenguaje integral: un salón en el que se privilegia la lectura. En R. Sáez Vega \& A. E. González Robles (Eds.). En voces de maestras: la transformación de prácticas educativas hacia la pedagogia del lenguaje integral (pp. 45-65). San Juan: Centro para el Estudio de la Lectura, la Escritura y la Literatura Infantil (CELELI).

Salgado, H. (2000). De la oralidad a la escritura. Propuesta didáctica para la construcción inicial de la lengua escrita. Buenos Aires: Editorial Magisterio del Río de la Plata.

Sastre-Gómez L. V., Celis-Leal, N. M., Roa de la Torre, J. D., \& Luengas-Monroy, C. F. (2017). La conciencia fonológica en contextos educativos y terapéuticos: efectos sobre el aprendizaje de la lectura. Educación y Educadores, 20(2), 175-190.

https://doi.org/10.5294/edu.2017.20.2.1

Sayago, Z., \& Chacón, M. (2006). Las prácticas profesionales en la formación docente: hacia un nuevo diario de ruta. Educere, 10(32), 55-66. http://r.issu. edu.do/l.php?l=137laR

Secretaría de Estado de Educación de la República Dominicana (1997). Ordenanza 05-1997. Santo Domingo: Secretaría de Estado de Educación de la República Dominicana.

http://r.issu.edu.do/l.php?l=138pvx

Secretaría de Estado de Educación de la República Dominicana (2003). Ordenanza 01-2004. Santo Domingo: Secretaría de Estado de Educación de la República Dominicana.

http://r.issu.edu.do/l.php?l=139aBL

Shanahan, T. (2015). Common Core State Standards: A New Role for Writing. Elementary School Journal, 115(4), 464-479.

https://doi.org/10.1086/681130

Sitko, B. M. (1998). Knowing how to write: metacognition in writing instruction. En D. J. Hacker, J. Dunlosky \& A. C. Graesser (Eds.). Metacognition in 
Educational Theory and Practice (pp. 93-116). London: Lawrence Erlbaum Associates.

Solé, I. (2009). Estrategias de lectura. Barcelona: Graó.

Stahl, K. A., Flanigan, K., \& McKenna, M. C. (2009). Assessment for Reading Instruction. New York: The Guilford Press.

Teberosky, A. (2000). Relectura de los sistemas de escritura en el desarrollo del niño. En F. Avendańo \& M. Baez (Eds.), Sistemas de escritura, constructivismo y educación (pp. 24-42). Buenos Aires: Homo Sapiens Ediciones.
Tunmer, W. E., \& Hoover, W. A. (2019). The cognitive foundations of learning to read: a framework for preventing and remediating reading difficulties. Australian Journal of Learning Difficulties, 24(1), 75-93. https://doi.org/10.1080/19404158.2019.1614081

UNICEF. (2010). Ecos de la revolución pingüina: Avances, debates y silencios en la reforma educacional. Chile: Universidad Nacional.

Wong-Fillmore, L., \& Snow, C. (2000). What teachers need to know about language. Washington, DC: ERIC Clearinghouse.

\section{CÓMO CITAR:}

Montenegro-de-Olloqui, L. (2020). Fortalezas y áreas de mejora de la formación inicial docente en lectoescritura en la República Dominicana. Revista Caribeña de Investigación Educativa (RECIE), 4(2), 23-45. https://doi.org/10.32541/recie.2020.v4i2. pp23-45 